\title{
UPR $^{\mathrm{mt}}$ regulation and output: a stress response mediated by mitochondrial-nuclear communication
}

\author{
Andrew Melber ${ }^{1}$, Cole M Haynes ${ }^{1}$ \\ ${ }^{I}$ Department of Molecular, Cell, and Cancer Biology, University of Massachusetts Medical School, Worcester, MA 01605, USA
}

The mitochondrial network is not only required for the production of energy, essential cofactors and amino acids, but also serves as a signaling hub for innate immune and apoptotic pathways. Multiple mechanisms have evolved to identify and combat mitochondrial dysfunction to maintain the health of the organism. One such pathway is the mitochondrial unfolded protein response $\left(\mathrm{UPR}^{\mathrm{mt}}\right)$, which is regulated by the mitochondrial import efficiency of the transcription factor ATFS-1 in C. elegans and potentially orthologous transcription factors in mammals (ATF4, ATF5, CHOP). Upon mitochondrial dysfunction, import of ATFS-1 into mitochondria is reduced, allowing it to be trafficked to the nucleus where it promotes the expression of genes that promote survival and recovery of the mitochondrial network. Here, we discuss recent findings underlying $U P R^{\mathrm{mt}}$ signal transduction and how this adaptive transcriptional response may interact with other mitochondrial stress response pathways.

Keywords: mitochondrial UPR; ISR; stress response; proteostasis

Cell Research (2018) 28:281-295. doi:10.1038/cr.2018.16; published online 9 February 2018

\section{Introduction}

When healthy, the highly dynamic and interconnected mitochondrial network provides the cell with energy in the form of ATP, cofactors such as heme and iron-sulfur clusters, amino acids, as well as nucleotides [1-3]. Mitochondria also serve as hubs for many signaling cascades including those regulating apoptosis and innate immunity [4-6]. During mitochondrial dysfunction many of these vitally important mitochondrial processes are compromised. With age, a notable increase in mitochondrial dysfunction occurs in otherwise healthy individuals and this decline is exacerbated in age-related neurological and cardiovascular diseases such as Parkinson's Disease and coronary artery disease, respectively [7-9]. The underlying causes of mitochondrial dysfunction in these scenarios include an accumulation of damaged mitochondrial genomes (mtDNA) that normally encode 13 essential oxidative phosphorylation (OXPHOS) components required for the function of respiratory complexes I, III and $\mathrm{IV}$, as well as the ATP synthase [10].

The remainder of the mitochondrial proteome is com-

Correspondence: Cole M Haynes

E-mail: cole.haynes@umassmed.edu prised of nuclear-encoded proteins ( $\sim 1500$ in humans) that are synthesized by cytosolic ribosomes and targeted to each compartment within the mitochondrial network and subsequently imported via the TOM (translocase of the outer membrane) and TIM (translocase of the inner membrane) channels $[11,12]$. Nuclear-encoded proteins are also susceptible to age-associated damage as they can become misfolded and aggregate [13], which can be exacerbated by locally produced reactive oxygen species (ROS) during OXPHOS-mediated ATP production [14]. Notably, mitochondrial defects are often pleiotropic. For example, OXPHOS or mitochondrial proteostasis perturbations reduce the rate of mitochondrial protein import by reducing the proton gradient or impairing mitochondrial chaperones, both of which must be maintained for efficient import [15].

\section{Cellular responses to mitochondrial dysfunction}

Organisms have evolved multiple mechanisms to recognize and resolve dysfunction within the mitochondrial network. Collectively, these mechanisms culminate with a response that recovers organelles that are salvageable and degrades organelles that are beyond repair, ultimately yielding a healthier mitochondrial network.

Severely damaged mitochondria are identified and 


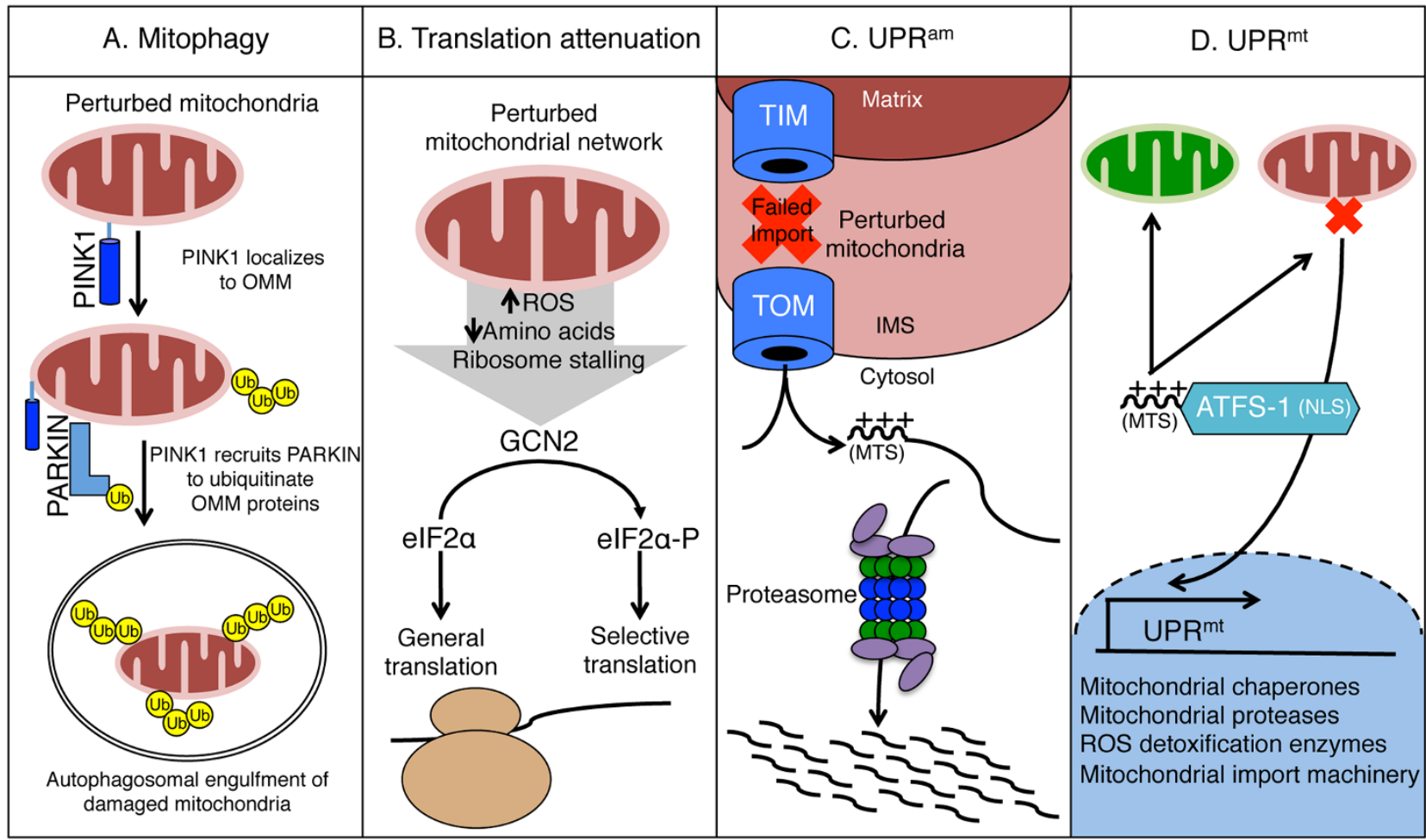

Figure 1 Mitochondrial stress response pathways. (A) Recognition and selective degradation of damaged mitochondria is mediated by mitophagy. The kinase PINK1 is stabilized specifically on damaged mitochondria where it recruits the ubiquitin ligase Parkin, which ubiquitinates multiple mitochondrial outer membrane proteins. Ubiquitinated mitochondria are then engulfed by autophagosomes and trafficked to lysosomes where they are degraded. (B) The kinase GCN2, which is activated during mitochondrial dysfunction, mediates translation attenuation during mitochondrial dysfunction by phosphorylating the translation initiation factor elF2 $\alpha$, which serves to reduce the influx of proteins into mitochondria. (C) Accumulation of mislocalized mitochondrial proteins in the cytosol stimulates proteasome activity to limit the accumulation of the toxic proteins in a pathway dubbed UPR ${ }^{a m}$ (unfolded protein response activated by mistargeted proteins). (D) The UPR ${ }^{\mathrm{mt}}$ is regulated by the competing organelle targeting sequences in the transcription factor ATFS-1. If ATFS-1 is imported into the mitochondrial matrix via the MTS, the transcription factor is degraded. However, if ATFS-1 cannot be imported due to mitochondrial dysfunction, it is trafficked to the nucleus, via the NLS, to activate transcription.

degraded via the process known as mitophagy (Figure 1A) [16]. Prior to the initiation of mitophagy, severely dysfunctional portions of the mitochondrial network are isolated through fission to prevent stress from diffusing throughout the entire network [17]. Mitophagy requires PINK1, a kinase that is imported into healthy mitochondria and ultimately degraded $[18,19]$. However, when mitochondrial import is perturbed, PINK1 is stabilized on the outer mitochondrial membrane (OMM) where it phosphorylates ubiquitin $[20,21]$. PINK1 also phosphorylates the ubiquitin ligase Parkin, recruiting it to the cytosolic face of the OMM where it poly-ubiquitinates multiple proteins [22-24]. Poly-ubiquitination serves to recruit machinery that engulfs the damaged organelle into an autophagosome, which is subsequently trafficked to lysosomes for degradation, thus ridding damaged compartments of the mitochondrial network $[25,26]$.
While mitophagy may represent a last resort for individual organelles, additional stress responses are in place to both limit the damage in defective mitochondria and facilitate the recovery of salvageable organelles. The vast majority of the mitochondrial proteome is synthesized on cytosolic ribosomes and imported into mitochondria, where each protein is processed and assembled with the help of mitochondrial-localized chaperones [12]. If not processed efficiently, the load of imported proteins can perturb mitochondrial proteostasis and impair essential mitochondrial activities. Cytosolic protein synthesis can be modulated during mitochondrial stress by the kinase GCN2, which phosphorylates the eukaryotic initiation factor alpha (eIF2 $\alpha$ ) [27], as a branch of the integrated stress response (ISR). GCN2 is likely stimulated by mitochondrial stress via reduced amino acids, increased ROS or ribosome stalling (Figure 1B) [28-30]. The 
phosphorylation of eIF $2 \alpha$ results in a decrease in protein synthesis, reducing the number of nascent peptides being imported into the mitochondria [31].

Reduced protein synthesis may also limit ribosome stalling during co-translational import into mitochondria. Recent work has demonstrated that Vms1, a protein that accumulates on damaged mitochondria by interacting with oxidized sterols $[32,33]$, resolves stalled ribosomes interacting with the TOM channel [34]. In the absence of Vms1, nascent protein fragments emerging from stalled mitochondrial-localized ribosomes are not accessible to the ubiquitin ligase Listerin due to the tight association with the TOM channel. Upon recruitment to dysfunctional mitochondria, Vms1 prevents the non-canonical addition of C-terminal alanine and threonine (CAT) tails to the nascent peptide as the mitochondrial matrix proteostasis machinery is unable to process CAT-tailed proteins for degradation. In sum, Vms1 localization to the outer membrane of damaged mitochondria prevents the aggregation of nascent protein fragments in mitochondria that can severely impair mitochondrial function.

A consequence of mitochondrial dysfunction is reduced protein import efficiency, which results in the accumulation of mislocalized mitochondrial proteins in the cytosol. A response, known as UPR ${ }^{\mathrm{am}}$ (UPR activated by protein mistargeting), promotes the degradation of the highly toxic mislocalized proteins by increasing proteasome activity and reducing protein synthesis (Figure 1C) $[35,36]$.

The focus of this review is on how cells regulate an adaptive transcriptional response during mitochondrial dysfunction to promote cell survival and recovery of the mitochondrial network known as the mitochondrial unfolded protein response $\left(\mathrm{UPR}^{\mathrm{mt}}\right)$. This pathway was initially discovered in mammalian cells, and further characterized in C. elegans [37]. The UPR ${ }^{\mathrm{mt}}$ is coordinated by multiple factors including the transcription factor ATFS-1 (Figure 1D). ATFS-1 is a transcription factor that promotes the expression of nuclear-encoded genes such as mitochondrial chaperones and proteases, ROS detoxification enzymes, and mitochondrial protein import components [38]. These induced proteins presumably enter functional and dysfunctional mitochondria in the cell to preserve function in healthy organelles and recover activity in damaged compartments. In addition to $\mathrm{UPR}^{\mathrm{mt}}$ regulation, the functional interactions between $U P R^{\mathrm{mt}}$ and translation attenuation, ribosome quality control and the $\mathrm{UPR}^{\mathrm{am}}$ will be discussed.

\section{Regulation and transcriptional outputs of the $U P R^{\mathrm{mt}}$}

$\mathrm{UPR}^{\mathrm{mt}}$ activation was first described in cultured mammalian cells exposed to ethidium bromide, which perturbs mitochondrial function by depleting mtDNA, resulting in the induction of transcripts encoding mitochondrial chaperones and proteases [39]. Similarly, overexpression of mutant ornithine transcarbamylase, lacking a segment required for proper processing and folding $(\triangle \mathrm{OTC})$, in the mitochondrial matrix elicited a similar transcriptional response, indicating a relationship linking mitochondrial function, proteostasis perturbations in mitochondria and UPR ${ }^{\mathrm{mt}}$ activation [37]. Subsequent work in C. elegans and mammalian systems has identified multiple components required for $\mathrm{UPR}^{\mathrm{mt}}$ activation, including sensors of mitochondrial dysfunction, regulators of mitochondrial-to-nuclear communication, chromatin regulators, and transcription factors.

\section{Perturbations that activate the UPR ${ }^{m t}$}

Numerous chemical, genetic and proteotoxic stresses have been shown to activate the UPR ${ }^{\mathrm{mt}}$, providing clues to the regulatory mechanisms and the physiologic or pathologic scenarios where the pathway may be important [39-41]. As mentioned above, the disruption of mitochondrial proteostasis by the expression of a mitochondrial-localized misfolded protein is capable of activating the UPR ${ }^{\mathrm{mt}}$ [37]. Presumably the misfolded proteins overwhelm the activity of mitochondrial chaperones in the matrix, which is essential for multiple mitochondrial activities including protein import. As such, the depletion of mitochondrial chaperones or proteases is also capable of activating the $\mathrm{UPR}^{\mathrm{mt}}[40]$.

The impairment of genes involved in diverse aspects of mitochondrial function also activates the UPR ${ }^{\mathrm{mt}}$, such as mitochondrial protein import (impairment of tim-23), OXPHOS (impairment of complex III or IV), coenzyme $\mathrm{Q}$ biogenesis (clk-1 inhibition), or lipid biogenesis (acl12 impairment) [28, 40, 42-44]. In addition, exposure to paraquat, a superoxide generator that perturbs respiratory chain function, causes $\mathrm{UPR}^{\mathrm{mt}}$ activation [42] as does the mitochondrial ribosome inhibitor doxycycline [45]. Importantly, all of these perturbations likely reduce mitochondrial import efficiency. By impairing TIM-23, an essential protein import component, transport into the matrix is directly impaired [42]. Respiratory chain perturbations potentially impair import by increasing ROS production and by depleting the proton gradient across the mitochondrial inner membrane. Recently, it has become appreciated that the expression of aggregate-prone proteins in the cytosol, such as mutant Huntingtin protein, linked to the onset of Huntington's disease, also activates the $\mathrm{UPR}^{\mathrm{mt}}$ [46]. How the disruption of proteostasis in the cytosol activates the UPR ${ }^{\mathrm{mt}}$ is unclear, however, it is clear that mitochondrial function is disrupted in 
diseases attributed to aggregate-prone proteins [7, 47].

Numerous studies suggest that UPR ${ }^{\mathrm{mt}}$ activation occurs in a variety of human diseases. Mitochondrial disease occurs in 1 of $\sim 3000$ individuals and assessment of these patients may provide direct insight into $\mathrm{UPR}^{\mathrm{mt}}$ function [48]. A cohort of patients with mitochondrial disease-associated myopathy (mitochondrial myopathy) correlated positively with an increase in $F G F-21$ [49], a gene known to be upregulated during mitochondrial stress where the UPR ${ }^{\mathrm{mt}}$ is activated $[50,51]$. Additionally, the upregulation of genes indicative of an activated UP$\mathrm{R}^{\mathrm{mt}}$ has been observed in mouse models of mitochondrial disease [52-54]. In addition to mitochondrial diseases, neurodegenerative conditions, including Alzheimer's disease (AD), are associated with mitochondrial dysfunction $[7,55]$. Recently, it has been shown in AD patient cohorts that an increase in the expression of UPR ${ }^{\mathrm{mt}}$-induced genes corresponded with increasing severity of the disease [56]. This includes induced UPR ${ }^{\mathrm{mt}}$ genes, such as the mitochondrial chaperone Hspd1 (Hsp60) and the mitochondrial protease Yme1L1 in brain tissue of $\mathrm{AD}$ patients [56, 57]. These studies in patients and disease models suggest a use for $\mathrm{UPR}^{\mathrm{mt}}$ genes as biomarkers for mitochondrial disease [49]. Furthermore, the different stressors are informative for our mechanistic understanding of $\mathrm{UPR}^{\mathrm{mt}}$ activation. Notably, many of the stressors that activate the $\mathrm{UPR}^{\mathrm{mt}}$ perturb mitochondrial protein import; this commonality between stressors provides mechanistic insight into the activation process.

\section{Coupling mitochondrial dysfunction to nuclear tran- scription}

In C. elegans, the UPR ${ }^{\mathrm{mt}}$ is regulated by the basic leucine zipper (bZIP) transcription factor ATFS-1, which contains both a mitochondrial targeting sequence (MTS) and a nuclear localization sequence (NLS) [58]. The presence of dual subcellular localization sequences enables the transcription factor to mediate mitochondrial-to-nuclear communication [42]. Under homeostatic conditions, ATFS- 1 is efficiently imported into the mitochondrial matrix and degraded by the protease LON. However, under mitochondrial dysfunction conditions, mitochondrial import of ATFS-1 is reduced, causing it to accumulate in the cytosol. Because ATFS-1 harbors a nuclear localization signal, it then traffics to the nucleus to activate the transcriptional response (Figure 2A). Thus, cells likely utilize mitochondrial import efficiency as an indicator of general mitochondrial function using ATFS1 as both a sensor and a mitochondria-to-nucleus signaling mechanism. Upon nuclear accumulation, ATFS-1 activates the transcription of over 500 genes that impact diverse cellular activities (Table 1) [38, 42, 59].
Once ATFS-1 is imported into the mitochondrial matrix, its MTS is cleaved and the remainder of the protein is degraded, suggesting that mitochondrial import efficiency is a key negative regulator of UPR ${ }^{\mathrm{mt}}$ activation [42]. Multiple studies have demonstrated that diverse forms of mitochondrial dysfunction reduce mitochondrial protein import efficiency $[18,35,42,60]$. The percentage of ATFS-1 that fails to be imported into mitochondria traffics to the nucleus and activates the nuclear transcriptional response [42]. In support of this model, mutations that cause amino acid substitutions within the MTS of ATFS-1 prevent the protein from being imported into the mitochondrial matrix, and result in constitutive $\mathrm{UPR}^{\mathrm{mt}}$ activation [61]. Thus, $\mathrm{UPR}^{\mathrm{mt}}$ activation occurs when the import efficiency of the mitochondrial network is reduced.

One aspect of the UPR ${ }^{\mathrm{mt}}$ that remains unclear is that if mitochondrial import of ATFS-1 is reduced, how are those gene products induced by ATFS-1 such as mitochondrial chaperones and proteases imported into the dysfunctional mitochondrial network? This issue is partially resolved by the ATFS-1-mediated induction of genes encoding components of the mitochondrial import complexes such as timm-17 and timm-23 [42]. In addition, import of $\mathrm{UPR}^{\mathrm{mt}}$-induced gene products is likely biased towards competent or healthier organelles (Figure 2A). However, this still does not address how or whether the $\mathrm{UPR}^{\mathrm{mt}}$ recovers dysfunctional mitochondria. One possibility relates to the strength of the MTS on ATFS-1 relative to those proteins induced during the $\mathrm{UPR}^{\mathrm{mt}}$ such as mitochondrial chaperones and proteases [62]. The program MitoFates analyzes amino acid composition, including net positive charge, to predict the likelihood that a specific amino terminal sequence will be imported into mitochondria [63]. Interestingly, MitoFates suggests that ATFS-1 has a significantly weaker MTS than the mitochondrial-targeted chaperones and proteases induced by ATFS-1 (Figure 2B). This comparison suggests that the relatively weak MTS on ATFS-1 may allow the transcription factor to serve as a sensor of mitochondrial import efficiency. While a percentage of ATFS-1 may fail to be imported into dysfunctional mitochondria, the strong MTSs on mitochondrial chaperones and proteases may permit import into dysfunctional mitochondria to re-establish proteostasis and promote organelle recovery.

\section{$U P R^{m t}$ activation requires mitochondrial stress-induced chromatin remodeling}

The importance of chromatin structure in the regulation of transcription is well established [64]. Interestingly, recent studies demonstrate that chromatin is specifically remodeled during mitochondrial dysfunc- 

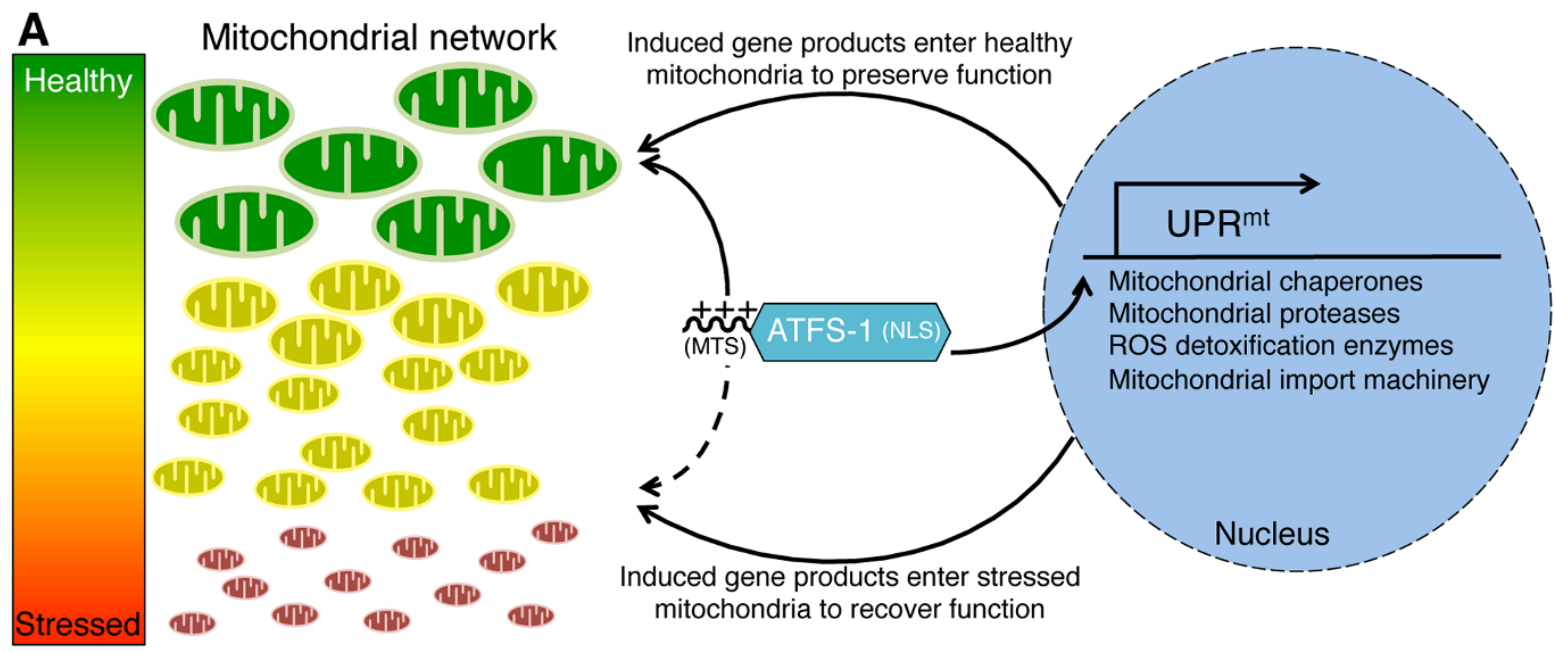

B

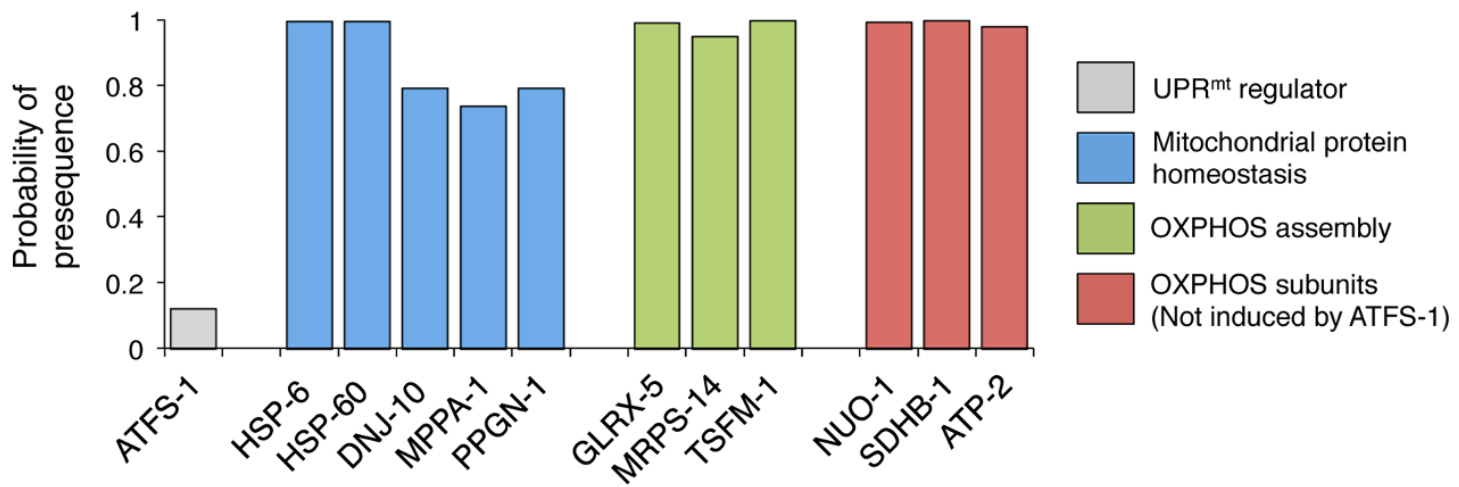

Figure 2 Model by which mitochondrial import efficiency of ATFS-1 and gene products induced by ATFS-1 promote mitochondrial network recovery. (A) The transcription factor ATFS-1 harbors both a mitochondrial targeting sequence (MTS) and a nuclear localization sequence (NLS). ATFS-1 is efficiently imported into healthy mitochondria (green), however, import efficiency is reduced by OXPHOS or mitochondrial proteostasis perturbations that cause mitochondrial dysfunction (yellow, red). If ATFS-1 fails to be imported into mitochondria, it is trafficked to the nucleus where it induces transcription of mitochondrial protective genes including mitochondrial chaperones and proteases, antioxidants as well as mitochondrial protein import components. In turn, mitochondrial import of the protective gene products promotes organelle stabilization and recovery. (B) Relative to the proteins induced during the UPR ${ }^{\mathrm{mt}}$, the program Mitofates [63] predicts that ATFS-1 has a substantially weaker mitochondrial signal sequence. We hypothesize that a weak MTS allows ATFS-1 to be sensitive to modest mitochondrial dysfunction and translocate to the nucleus. In turn, the strong MTSs in those proteins induced by ATFS-1 can still enter dysfunctional mitochondria with reduced import efficiency to recover function. HSP-6, HSP-60 and DNJ-10 are mitochondrial chaperones, MPPA-1 is a subunit of the mitochondrial presequence processing protease, PPGN-1 is a matrix-localized protease, GLRX-5 is a glutaredoxin that functions in mitochondrial iron-sulfur cluster biogenesis, MRPS-14 is a subunit of the mitochondrial ribosome, TSFM-1 is a mitochondrial translational elongation factor, all of which are induced during mitochondrial dysfunction by ATFS-1. NUO-1, SDHB-1 and ATP-2 are all subunits of the OXPHOS complexes, none of which are activated by the UPR ${ }^{\mathrm{mt}}$ (complexes I, II and V, respectively).

tion to promote $\mathrm{UPR}^{\mathrm{mt}}$ activation $[65,66]$. The histone methyltransferase, MET-2 in concert with LIN-65 [65], along with two jumonji domain histone demethylases, JMJD-3.1 and JMJD-1.2 [66], were recently found to be required for $\mathrm{UPR}^{\mathrm{mt}}$ activation. Both the histone methyltransferase and histone demethylase activities are stimulated by mitochondrial dysfunction. Interestingly, MET-
2 and LIN-65 promote global chromatin condensation, whereas the histone demethylases maintain the promoters of $\mathrm{UPR}^{\mathrm{mt}}$-induced genes in an open or transcriptionally competent state. This chromatin state is further stabilized by the homeobox protein DVE-1 and ubiquitin-like protein UBL-5, both of which are also required for UP$\mathrm{R}^{\mathrm{mt}}$ activation $[65,67,68]$. Interestingly, JMJD-3.1 and 
Table 1 Genes induced during $\mathrm{UPR}^{\mathrm{mt}}$ in both $C$. elegans and mammalian models

\begin{tabular}{|c|c|c|c|}
\hline \multicolumn{4}{|c|}{ C. elegans regulated genes } \\
\hline & Gene & Function & References \\
\hline \multirow[t]{3}{*}{ Mitochondrial protein homeostasis } & $d n j-10$ & Mitochondrial DnaJ, protein chaperone & {$[38,42,59]$} \\
\hline & ppgn-1 & Paraplegin AAA protease (mitochondrial) & {$[38,59]$} \\
\hline & ymel-1 & Mitochondrial AAA protease & {$[38,42]$} \\
\hline \multirow[t]{2}{*}{ Mitochondrial dynamics } & $d r p-1$ & Dynamin-related protein, mitochondrial fission & {$[38,42,59]$} \\
\hline & $m f f-2$ & Mitochondrial fission factor & {$[38,42,59]$} \\
\hline \multirow[t]{2}{*}{ Innate immunity } & $a b f-2$ & Antimicrobial peptide & {$[42,104]$} \\
\hline & lys-2 & Secreted lysosome & {$[42,104]$} \\
\hline \multirow{2}{*}{ Metabolism } & $c l k-1$ & Coenzyme Q biosynthesis & {$[38,42,59]$} \\
\hline & $l d h-2$ & Lactate dehydrogenase & {$[38,42]$} \\
\hline \multicolumn{4}{|c|}{ Mammalian regulated genes } \\
\hline & Gene & Function & References \\
\hline \multirow[t]{4}{*}{ ATF4-regulated } & ATF5 & Transcription factor, $\mathrm{UPR}^{\mathrm{mt}}$ regulator & {$[72]$} \\
\hline & CHOP & Transcription factor, $\mathrm{UPR}^{\mathrm{mt}}$ regulator & {$[71,72]$} \\
\hline & FGF21 & Fibroblast growth factor, mitokine & {$[50,51]$} \\
\hline & ASNS & Asparagine synthetase & {$[51]$} \\
\hline ATF5-regulated & $\mathrm{mtHSP70}$ & Mitochondrial chaperone & {$[73]$} \\
\hline
\end{tabular}

JMJD-1.2 are both necessary and sufficient for UPR ${ }^{\mathrm{mt}}$ activation and stimulated during mitochondrial dysfunction in a manner independent of ATFS-1 [66]. Combined, these findings demonstrate the requirement for at least two inputs to mediate UPR ${ }^{\mathrm{mt}}$ activation presumably to appropriately match $\mathrm{UPR}^{\mathrm{mt}}$ outputs or strength of activation to related aspects of animal physiology such as development and aging.

\section{$U P R^{m t}$ regulation via inter-cellular communication}

In addition to the cell-autonomous $\mathrm{UPR}^{\mathrm{mt}}$ regulation discussed in previous sections, UPR ${ }^{\mathrm{mt}}$ activation can be communicated between cells and tissues via endocrine signaling. Cell-non-autonomous UPR ${ }^{\mathrm{mt}}$ activation has been described using multiple neuronal-specific mitochondrial stressors, which causes intestinal UPR ${ }^{\mathrm{mt}}$ activation [43]. The expression of an aggregate-prone mutant Huntingtin protein in neurons is capable of inducing the $U P R^{\mathrm{mt}}$ elsewhere in the organism, which requires serotonin secretion [46]. Similarly, disruption of mitochondrial proteostasis specifically in neurons by utilizing
CRISPR/Cas9 to impair the protease SPG-7 also resulted in intestinal $\mathrm{UPR}^{\mathrm{mt}}$ activation [69]. This approach led to the discovery of a second secreted factor, the neuropeptide FLP-2, and a neuronal circuit as being required for cell non-autonomous signaling. Endocrine- or mitokine-regulated activation of the UPR ${ }^{\mathrm{mt}}$ likely serves to coordinate activation between tissues, potentially as an early warning system linking sensory neurons that prime a defense for a future mitochondrial stress in distal tissues.

\section{Mammalian $U P R^{m t}$ regulation}

While the initial discovery of the UPR ${ }^{\mathrm{mt}}$ was made in cultured mammalian cells [39], many of the genes required for UPR ${ }^{\mathrm{mt}}$ activation were identified in C. elegans, owing to the relative ease of using the organism to perform genetic screens $[67,70]$. Interestingly, numerous recent studies in mammalian systems have suggested considerable conceptual and mechanistic overlap between $\mathrm{UPR}^{\mathrm{mt}}$ signaling in the two systems although added layers of regulation likely exist in mammals [71-73]. For 
example, a functional ortholog of ATFS-1 was recently discovered. ATF5 is a bZIP transcription factor regulated by mitochondrial import efficiency similarly to ATFS1. Importantly, ATF5 expression is capable of restoring $\mathrm{UPR}^{\mathrm{mt}}$ activation when expressed in nematodes lacking ATFS-1. Furthermore, in cultured cells ATF5 promoted OXPHOS and cell growth during mitochondrial dysfunction by inducing expression of several mitochondrial chaperone and protease genes [73].

In addition to ATF5, at least two other bZIP transcrip- tion factors, ATF4 and CHOP, are also involved in $\mathrm{UPR}^{\mathrm{mt}}$ activation [72, 74-77]. The relationship between ATF4, CHOP and ATF5 during mitochondrial dysfunction remains to be determined. However, it is clear that the expression of all three transcription factors requires the ISR $[78,79]$. Hence, the activation of the ISR is necessary for $\mathrm{UPR}^{\mathrm{mt}}$ activation in mammals. ISR activation is mediated by four kinases that phosphorylate eIF $2 \alpha$ in response to specific stresses (Figure 3). The ISR kinase PERK responds to unfolded protein accumulation in the endoplas-

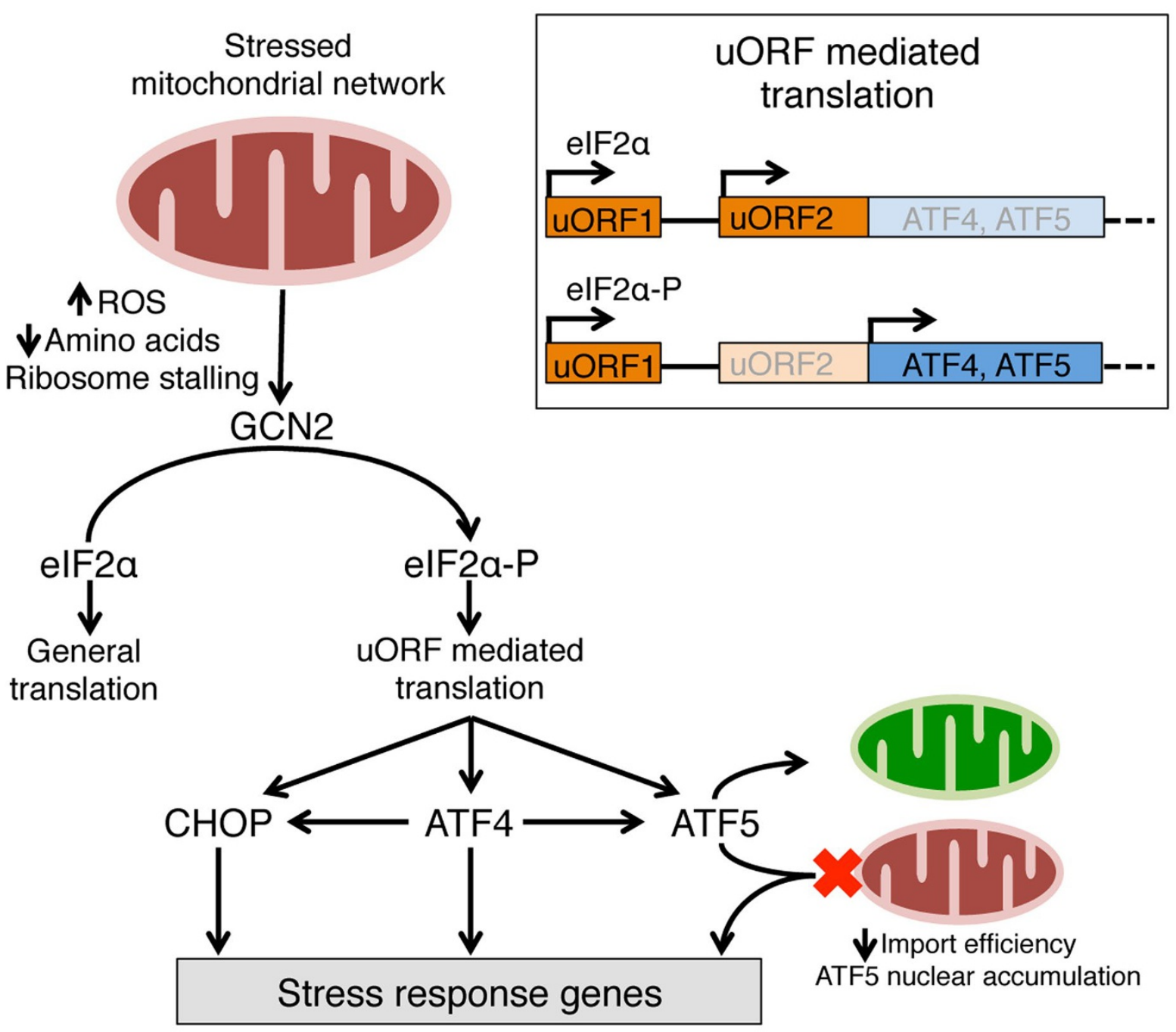

Figure 3 The mammalian UPR ${ }^{\mathrm{mt}}$ is intimately associated with the integrated stress response (ISR). During mitochondrial dysfunction, the translation initiation factor elF $2 \alpha$ is phosphorylated by one of four elF2 $\alpha$-specific kinases such as GCN2 (also PERK, PKR and HRI). elF2 $\alpha$ phosphorylation results in reduced protein synthesis with a concomitant increase in translation of those mRNAs harboring uORFs in the 5' UTR. The mRNAs encoding the transcription factors CHOP, ATF4 and ATF5 all harbor multiple uORFs and are preferentially translated during mitochondrial dysfunction (inset). While the precise relationship between these three transcription factors remains to be determined during mitochondrial stress, all three are required for the induction of genes associated with the UPR ${ }^{\mathrm{mt}}$. Both CHOP and ATF4 induce transcription of Atf5. Like ATFS-1 in C. elegans, ATF5 harbors a mitochondrial targeting sequence potentially allowing it to specifically respond to mitochondrial stress via reduced mitochondrial protein import efficiency. 
mic reticulum, PKR responds to cytosolic double stranded RNA, and HRI is activated by heme depletion [80]. GCN2 is activated by mitochondrial stress as well as by amino acid depletion, ROS and ribosome stalling [28, 81]. Phosphorylation of the translation initiation factor eIF $2 \alpha$ results in reduced protein synthesis, but preferential synthesis of mRNAs harboring small upstream open reading frames (uORFs) in the $5^{\prime}$ untranslated region (UTR).

Selective translation mediated by eIF $2 \alpha$ phosphorylation requires one or more uORFs upstream of a primary open reading frame (ORF) in the 5' UTR (Figure 3, inset). Following translation of the first uORF, the ribosome dissociates and the $40 \mathrm{~S}$ subunit continues to scan the mRNA for the next ORF. In the absence of eIF $2 \alpha$ phosphorylation, translation re-initiation occurs quickly resulting in translation of the second uORF, which overlaps the translational start site (methionine) of the primary ORF, preventing translation. However, if eIF $2 \alpha$ is phosphorylated, regeneration of the initiation complex is slowed, allowing the ribosome to scan through the start codon of the second UORF, thus enabling the ribosome to engage the primary ORF at a higher rate (Figure 3 inset) [81-83]. ATF4, CHOP and ATF5 are three such proteins that require eIF $2 \alpha$ phosphorylation to be synthesized due to the presence of uORFs in the $5^{\prime}$ UTR of the mRNA encoding each protein $[78,79,84,85]$.

Thus, in mammals, $\mathrm{UPR}^{\mathrm{mt}}$ activation requires eIF $2 \alpha$ phosphorylation and is intimately associated with the ISR. However, in nematodes the UPR ${ }^{\mathrm{mt}}$ does not require eIF2 $\alpha$ kinases (GCN2) or eIF2 $\alpha$ phosphorylation; thus, the transcriptional response functions in parallel to the regulation of translation [28]. The translational attenuation likely complements the transcriptional response by reducing the nascent protein load in the matrix, so that $\mathrm{UPR}^{\mathrm{mt}}$-induced chaperones and proteases may better promote proteostasis in the mitochondria. In mammals, translation attenuation is required for the transcriptional response to mitochondrial dysfunction.

Beyond all requiring eIF $2 \alpha$ phosphorylation, the functional relationship between ATF4, CHOP and ATF5 during mitochondrial stress remains unclear. One possibility is that transcription of ATF5 is regulated by both ATF4 and CHOP, which has been shown previously, but not in the context of mitochondrial stress $[78,79]$. Once expressed, ATF5 can subsequently activate mitochondrial-specific stress response genes if the mitochondrial import of ATF5 is reduced resulting in the nuclear localization of the transcription factor (Figure 3) [73]. However, CHOP and ATF4 likely contribute to transcriptional adaptations to mitochondrial stress directly as well.

Surprisingly, recent work has implicated mTORC1 (mechanistic target of rapamycin complex 1) in $\mathrm{UPR}^{\mathrm{mt}}$ regulation also via uORF-mediated translation regulation. mTORC1 is a kinase that regulates cellular growth in response to cellular nutrition, ATP depletion as well as growth factors [86]. mTORC1 activation stimulates protein synthesis by phosphorylating S6 kinase [87], and impaired mTORC1 results in reduced protein synthesis which coincides with increased autophagy [88, 89]. Interestingly, the affected tissues in a mouse model of mitochondrial myopathy driven by the accumulation of deleterious mtDNAs, had increased mTORC1 activation and ATF4 activity [54]. While it is unclear how increased mTORC1 activity promotes ATF4 synthesis, it does require uORFs [90]. mTORC1 activation in mitochondrial myopathy also activated ATF5 along with multiple metabolic genes (see below), which were impaired by treatment with rapamycin, demonstrating a requirement for mTORC1 signaling. This work raises a number of exciting questions including how mTORC1 is stimulated during mitochondrial dysfunction and how or whether mTORC1 interfaces with the ISR to regulate the UPR ${ }^{\mathrm{mt}}$ ?

\section{Metabolic adaptations during mitochondrial stress}

As mitochondria play a central role in metabolism by producing ATP, amino acids, lipids and nucleic acids $[2,3]$, it is perhaps not surprising that the expression of many metabolic genes is altered during mitochondrial dysfunction via the UPR $^{\mathrm{mt}}$ [42]. For example, ATFS-1 binds the promoters of all glycolysis genes driving their induction during mitochondrial stress presumably to allow the cell to maintain ATP levels, independent of mitochondrial function [38]. Alternatively, ATFS-1 binds the promoters of many TCA cycle and OXPHOS genes, but represses or limits their transcription during mitochondrial stress [38]. Reducing the rate of OXPHOS complex biogenesis while maintaining basal metabolic function through glycolysis may be protective by (1) restricting the amount of ROS byproducts, (2) reducing the load of unassembled OXPHOS components in mitochondria where proteostasis is perturbed, and (3) to reestablish the stoichiometric balance of mitochondrial- and nuclear-encoded OXPHOS subunits.

Metabolic adaption to mitochondrial stress has also been observed in mammalian systems. For example, OXPHOS-deficient cells isolated from mitochondrial disease patients have been shown to survive in culture by increasing glycolysis [91]. In addition, mammalian models of mitochondrial stress have shown that ATF4 promotes one-carbon metabolism [72, 92]. One-carbon metabolism is a general metabolic process providing single-carbon units for an array of biosynthetic processes including nu- 
cleotide and amino acid biosynthesis [93]. Furthermore, mTORC1, noted above for its role in $\mathrm{UPR}^{\mathrm{mt}}$ activation, promotes purine synthesis [90]. While there is an immediate benefit for maintaining cellular function through metabolic alterations, the long-term implications of a constitutively active $\mathrm{UPR}^{\mathrm{mt}}$ that promotes glycolysis and biosynthetic processes may be detrimental as this shift is characteristic of highly proliferative cells.

\section{Physiologic roles of mitochondrial stress response}

\section{Mitochondrial stress responses during aging}

A decline in mitochondrial function with age has been well documented in model systems including yeast, worms, flies and mice as well as in tissues isolated from patients [7, 94-97]. The overall decline in mitochondrial function is highlighted by reduced oxygen consumption with a corresponding reduction in respiratory complex activity $[94,95]$. Notably this decline has been attributed to the onset of many age-related physiological disorders such as Parkinson's disease and coronary artery disease [7-9].

One of the first described physiologic roles of the UP$\mathrm{R}^{\mathrm{mt}}$ was during the lifespan extension caused by modest perturbations in OXPHOS. Mutations that perturb OXPHOS or coenzyme Q biogenesis in nematodes, flies and mice have increased longevity [98-102]. The mitochondrial perturbations that lead to the extension of lifespan also cause activation of the UPR ${ }^{\mathrm{mt}}$ [43]. Importantly, the development and increased longevity of these animals requires multiple UPR ${ }^{\mathrm{mt}}$ components, such as jmjd-3.1, $h a f-1$ and $d v e-1$, demonstrating that the pathway is protective during mitochondrial dysfunction $[65,66,100$, $103,104]$. Furthermore, $\mathrm{UPR}^{\mathrm{mt}}$ activation by neuronal overexpression of the Jumonji histone demethylase is sufficient to extend worm lifespan [66]. However, which $\mathrm{UPR}^{\mathrm{mt}}$ outputs contribute to development and longevity remains unclear.

Along with UPR ${ }^{\mathrm{mt}}$ activation [43], mitophagy has also been shown to decline with age [105]. Impressively similar to increased UPR ${ }^{\mathrm{mt}}$ activation, increased mitophagy, which is regulated by PINK1 and Parkin, as well as the bZIP transcription factor SKN-1 (Nrf2 in mammals), also prolongs worm lifespan [106-108]. SKN-1 is induced by ATFS-1 during mitochondrial stress as are other mitophagy components [42], suggesting some degree of coordination between mitophagy and the UPR ${ }^{\mathrm{mt}}$ during aging, but the precise relationship remains to be determined.

\section{The UPR $R^{m t}$ in aging stem cells}

The role of the UPR ${ }^{\mathrm{mt}}$ in longevity has primarily been examined in C. elegans, an organism that lacks somatic stem cells. Thus, unlike mammals, worms are unable to regenerate or replace cells in somatic tissues. Importantly, the ability to regenerate cell types declines during aging due to reduced stem cell function [109-111]. Maintenance of the stem cell pool relies on a balance between self-renewal, periods of quiescence, and differentiation [112-114]. Mitochondria are relatively inactive in quiescent stem cells, which primarily rely on glycolysis for ATP production. However, upon differentiation mitochondrial biogenesis occurs, which is associated with an increase in OXPHOS $[115,116]$. Numerous reports have linked mitochondrial dysfunction with the decline in stem cell function during aging [116-118]. For example, transgenic mice that accumulate mtDNA mutations due to the expression of an mtDNA polymerase lacking proofreading activity age prematurely and exhibit stem cell dysfunction [117-121].

Recent work has suggested a role of the UPR ${ }^{\mathrm{mt}}$ and the sirtuin SIRT7 in maintaining hematopoietic stem cell (HSC) function during aging [122]. During mitochondrial stress, SIRT7 is transcriptionally induced and binds to the transcription factor nuclear respiratory factor 1 (NRF1), which induces transcripts required for mitochondria biogenesis including mitochondrial ribosome components. Importantly, SIRT7 represses NRF1's transcription activity. Thus, HSCs lacking SIRT7 have a higher degree of mitochondrial biogenesis and mitochondrial stress, consistent with increased mitochondrial chaperone and protease transcription. Combined, this study suggests that SIRT7 serves to re-establish mitochondrial proteostasis by reducing the load of newly synthesized mitochondrial proteins. Interestingly, SIRT7-deficient HSCs are prone to aberrant differentiation. Thus, SIRT7 represses mitochondrial biogenesis and promotes quiescence to maintain the HSC pool. As the levels of SIRT7 decrease in aged HSCs, the deregulation of the $\mathrm{UPR}^{\mathrm{mt}}$ may contribute to HSC dysfunction during aging [122]. It remains to be determined whether ATF4, ATF5 or CHOP regulates induction of SIRT7.

\section{Mitochondrial stress responses contribute to deleterious mtDNA propagation}

The mitochondrial genome (mtDNA) encodes 13 essential OXPHOS components, 2 ribosomal RNAs, and 22 tRNAs required for mRNA translation in the mitochondrial matrix [10]. Most metazoan cells harbor hundreds of copies of mtDNA with 5-10 per organelle [123, 124]. Steady-state levels of mtDNA are maintained by mitochondrial biogenesis which includes mtDNA replication, requiring the mitochondrial DNA polymerase and TFAM, a protein that packages mtDNA into nucleoids [125]. In addition, mitophagy affects steady-state mtD- 
NA levels by degrading severely damaged mitochondria [125].

Given the high number of mtDNAs per cell, a low percentage of deleterious mtDNAs $(\triangle \mathrm{mtDNA}$; mutations or large deletions) is well tolerated, presumably due to the high percentage of wild-type mtDNAs [126]. In fact, deep sequencing studies have shown that $\triangle \mathrm{mtDNAs}$ are found in most individuals [127, 128]. However, as cells and organisms age, an individual $\triangle$ mtDNA can accumulate to a point that perturbs OXPHOS, which impairs cellular function [127]. The accumulation of $\triangle \mathrm{mtDNAs}$ likely contributes to the reduction in mitochondrial function that occurs in aging cells such as neurons or muscles, as well as in cancer cells $[129,130]$. The underlying mechanisms that impact $\triangle$ mtDNA dynamics remain unclear, but recent work suggests antagonistic roles for the $\mathrm{UPR}^{\mathrm{mt}}$ and mitophagy.

Perhaps not surprisingly, in a C. elegans model of deleterious heteroplasmy (cells containing both wildtype mtDNA and $\triangle \mathrm{mtDNA}$ ) the $\mathrm{UPR}^{\mathrm{mt}}$ is activated, as the $\triangle \mathrm{mtDNA}$ lacks the genes required for the expression of several OXPHOS subunits $[59,131]$. In addition to reduced OXPHOS activity, the heteroplasmic worms displayed mitonuclear protein imbalance as the OXPHOS complex-encoding mRNAs expressed from both wildtype mtDNAs and $\triangle \mathrm{mtDNAs}$ were overexpressed relative to nuclear-encoded subunits, suggesting additional proteostasis perturbations [59, 103, 131]. Presumably, $\mathrm{UPR}^{\mathrm{mt}}$ activation occurs in an attempt to maintain proteostasis and promote recovery of mitochondrial dysfunction, similar to what occurs when the mitochondrial dysfunction is caused by a toxin or a mutation within a nuclear-encoded OXPHOS subunit. Surprisingly, deletion of ATFS-1 resulted in a preferential reduction in $\triangle$ mtDNA, with a concomitant increase in wild-type mtDNAs. Furthermore, constitutive activation of the $\mathrm{UPR}^{\mathrm{mt}}$ in the heteroplasmic worm was sufficient to cause increased mitochondrial biogenesis, which resulted in a preferential increase of $\triangle \mathrm{mtDNAs}$ relative to wild-type mtDNAs $[59,131]$. Combined, these data demonstrate that a potential consequence of $\mathrm{UPR}^{\mathrm{mt}}$ activation is the propagation of $\triangle m t D N A s$, however, it remains unclear how the $\triangle$ mtDNA outcompetes wild-type mtDNA.

In contrast to the UPR ${ }^{\mathrm{mt}}$, multiple studies have demonstrated a role for mitophagy in limiting the accumulation of $\triangle$ mtDNAs [59, 131-133]. Presumably, the organelles in which the $\triangle \mathrm{mtDNA} / \mathrm{mtDNA}$ ratio severely impairs OXPHOS are recognized by PINK1 and degraded via mitophagy. However, those organelles that contain a low percentage of $\triangle \mathrm{mtDNAs}$ and are able to maintain OXPHOS avoid mitophagy. One possible mechanism by which the $\mathrm{UPR}^{\mathrm{mt}}$ maintains $\triangle \mathrm{mtDNAs}$ is simply by maintaining mitochondrial proteostasis and function, thus limiting the detection and degradation of mitochondria harboring $\triangle \mathrm{mtDNAs}$ by mitophagy. Consistent with this model, when ATFS-1 is impaired in the absence of Parkin, $\triangle$ mtDNAs are depleted but not to the levels observed when mitophagy is intact $[59,131]$.

Conceptually similar results were recently demonstrated in a mouse model of mitochondrial myopathy caused by expression of a mutant version of the mtDNA replicative helicase Twinkle, which causes aberrant accumulation of $\Delta$ mtDNAs $[54,134]$. The mice accumulate a variety of $\triangle \mathrm{mtDNAs}$ in post-mitotic cells, leading to muscle OXPHOS deficiency. As discussed above, it was demonstrated that the UPR ${ }^{\mathrm{mt}}$ was induced in these mice in an mTORC1-dependent manner. Impressively, mTORC1 inhibition resulted in reduced $\mathrm{UPR}^{\mathrm{mt}}$ activation and limited the accumulation of $\triangle$ mtDNAs, which reduced the progression of mitochondrial myopathy. These data also suggest that $\triangle$ mtDNA propagation or accumulation is associated with mitochondrial biogenesis [54]. Interestingly, in addition to limiting mitochondrial biogenesis, inhibition of mTORC1 also activates mitophagy at a higher rate in cells containing heteroplasmic $\triangle \mathrm{mtDNA}[135]$, suggesting a second mechanism by which the UPR ${ }^{\mathrm{mt}}$ may antagonize mitophagy and promote the accumulation of $\Delta$ mtDNAs.

\section{Perspective}

In this review, we have focused on the role of the $\mathrm{UPR}^{\mathrm{mt}}$ in maintaining the mitochondrial network in model systems as well as in disease and disease models. While it is clear that multiple pathways respond to mitochondrial dysfunction and contribute to mitochondrial network homeostasis, data suggesting interactions between the UP$\mathrm{R}^{\mathrm{mt}}$, mitophagy, translation modulation and proteasome function are only beginning to emerge. For example, these responses are activated in response to similar forms of mitochondrial stress such as $\triangle$ mtDNA heteroplasmy or exposure to toxins such as paraquat $[131,136]$. Furthermore, numerous reports demonstrate increased eIF $2 \alpha$ phosphorylation and ISR activation during mitochondrial dysfunction, which not only reduces protein synthesis, but is also required for the preferential synthesis of ATF4, CHOP and ATF5 that promote recovery of mitochondrial network function by adapting transcription [72, $78,79,84]$. These findings make it clear that the UPR ${ }^{\mathrm{mt}}$ in mammals is included within the relatively broad ISR requiring eIF2 $\alpha$ phosphorylation. However, the mechanism by which the ISR is specified to respond specifically to mitochondrial stress remains unclear.

Mitochondrial protein import efficiency is a central 
component common to the regulation of mitophagy, the $\mathrm{UPR}^{\mathrm{mt}}$ and proteasome stimulation. Impaired import causes ATFS-1 and ATF5 to traffic to the nucleus to activate the UPR ${ }^{\mathrm{mt}}$, and PINK1 to accumulate in the mitochondrial outer membrane to initiate mitophagy, and causes the accumulation of mislocalized mitochondrial proteins in the cytosol that stimulates proteasome activity to maintain cytosolic proteostasis. While OXPHOS and mitochondrial proteostasis are often perturbed by conditions that activate these pathways, it is currently unclear whether more direct regulation of mitochondrial import efficiency plays a role in coordinating these pathways. Recent work has demonstrated that TOM complex-mediated import is regulated by casein kinase 2 (CK2) and protein kinases A (PKA) in yeast. However, whether TOM or either kinase is regulated during mitochondrial stress is unknown [137-139].

Considerable data indicate that $\mathrm{UPR}^{\mathrm{mt}}$ activation provides protection during mitochondrial dysfunction; however, there are also negative consequences of prolonged or dysregulated UPR ${ }^{\mathrm{mt}}$ activation that occurs in the context of deleterious heteroplasmy. It is clear that UPR ${ }^{\mathrm{mt}}$ activation promotes development and extends lifespan during mild mitochondrial dysfunction, suggesting that approaches to enhance UPR ${ }^{\mathrm{mt}}$ activation may be useful therapeutics. However, several recent reports demonstrate that prolonged $\mathrm{UPR}^{\mathrm{mt}}$ activation can exacerbate mitochondrial dysfunction caused by deleterious mtDNA accumulation $[54,59,131]$. In the context of deleterious heteroplasmy, approaches to impair or limit $\mathrm{UPR}^{\mathrm{mt}}$ activation improve mitochondrial function. Recent work in mice demonstrates that $\mathrm{UPR}^{\mathrm{mt}}$ inhibition can be achieved by treatment with rapamycin, providing optimism for future therapeutic approaches. The authors found that mitochondrial dysfunction in mouse muscle cells caused by mtDNA damage resulted in increased mTORC1 activation that was required for increased $\mathrm{UPR}^{\mathrm{mt}}$ activation. Importantly, treatment of mice with the mTORC1 inhibitor rapamycin reduced $\mathrm{UPR}^{\mathrm{mt}}$ activation, slowing mitochondrial myopathy progression caused by the accumulation of deleterious mtDNAs [54].

Here, we have reviewed the progress made in understanding how cells and organisms evaluate and respond to dysfunction in the mitochondrial network and adapt transcription accordingly. Included in the $\mathrm{UPR}^{\mathrm{mt}}$ are not only transcripts that promote mitochondrial proteostasis and mitochondrial biogenesis, but also metabolic adaptations that promote survival and network recovery. While a number of conserved regulatory components required to signal the UPR ${ }^{\mathrm{mt}}$ and their individual functions have been identified, their precise interactions remain to be determined. Furthermore, "mitochondrial dysfunction" likely encompasses diverse physiological scenarios; how the $\mathrm{UPR}^{\mathrm{mt}}$ is specified or receives inputs during each scenario remains to be elucidated. Considering the recent pace of $U_{P R}{ }^{\text {mt }}$-related discoveries, we are optimistic that the answers to these questions and more will be resolved.

\section{Acknowledgments}

This work was supported by NIH fellowship 5T32CA130807 to AM and the Mallinckrodt Foundation, HHMI and NIH grants R01AG040061, R01AG047182 and R01HL127891 to CMH.

\section{References}

1 Lill R, Hoffmann B, Molik S, et al. The role of mitochondria in cellular iron-sulfur protein biogenesis and iron metabolism. Biochim Biophys Acta 2012; 1823:1491-1508.

2 Wang L. Mitochondrial purine and pyrimidine metabolism and beyond. Nucleosides Nucleotides Nucleic Acids 2016; 35:578-594.

3 Wellen KE, Thompson CB. A two-way street: reciprocal regulation of metabolism and signalling. Nat Rev Mol Cell Biol 2012; 13:270-276.

4 Bohovych I, Khalimonchuk O. Sending out an SOS: Mitochondria as a signaling hub. Front Cell Dev Biol 2016; 4:109.

5 Weinberg SE, Sena LA, Chandel NS. Mitochondria in the regulation of innate and adaptive immunity. Immunity 2015; 42:406-417.

6 Bhola PD, Letai A. Mitochondria-judges and executioners of cell death sentences. Mol Cell 2016; 61:695-704.

7 Duarte JM, Schuck PF, Wenk GL, Ferreira GC. Metabolic disturbances in diseases with neurological involvement. Aging Dis 2014; 5:238-255.

8 Ballinger SW. Mitochondrial dysfunction in cardiovascular disease. Free Radic Biol Med 2005; 38:1278-1295.

9 Krzywanski DM, Moellering DR, Fetterman JL, Dunham-Snary KJ, Sammy MJ, Ballinger SW. The mitochondrial paradigm for cardiovascular disease susceptibility and cellular function: a complementary concept to Mendelian genetics. Lab Invest 2011; 91:1122-1135.

10 Anderson S, Bankier AT, Barrell BG, et al. Sequence and organization of the human mitochondrial genome. Nature 1981; 290:457-465.

11 Prokisch H, Scharfe C, Camp DG 2nd, et al. Integrative analysis of the mitochondrial proteome in yeast. PLoS Biol 2004; 2:e160.

12 Chacinska A, Koehler CM, Milenkovic D, Lithgow T, Pfanner N. Importing mitochondrial proteins: machineries and mechanisms. Cell 2009; 138:628-644.

13 David DC, Ollikainen N, Trinidad JC, Cary MP, Burlingame $\mathrm{AL}$, Kenyon C. Widespread protein aggregation as an inherent part of aging in C. elegans. PLoS Biol 2010; 8:e1000450.

14 Murphy MP. How mitochondria produce reactive oxygen species. Biochem J 2009; 417:1-13.

15 MacKenzie JA, Payne RM. Mitochondrial protein import and human health and disease. Biochim Biophys Acta 2007; 1772:509-523.

16 Ashrafi G, Schwarz TL. The pathways of mitophagy for qual- 
ity control and clearance of mitochondria. Cell Death Differ 2013; 20:31-42.

17 Twig G, Elorza A, Molina AJ, et al. Fission and selective fusion govern mitochondrial segregation and elimination by autophagy. EMBO J 2008; 27:433-446.

18 Narendra DP, Jin SM, Tanaka A, et al. PINK1 is selectively stabilized on impaired mitochondria to activate Parkin. PLoS Biol 2010; 8:e1000298.

19 Valente EM, Abou-Sleiman PM, Caputo V, et al. Hereditary early-onset Parkinson's disease caused by mutations in PINK1. Science 2004; 304:1158-1160.

20 Kane LA, Lazarou M, Fogel AI, et al. PINK1 phosphorylates ubiquitin to activate Parkin E3 ubiquitin ligase activity. J Cell Biol 2014; 205:143-153.

21 Koyano F, Okatsu K, Kosako H, et al. Ubiquitin is phosphorylated by PINK1 to activate parkin. Nature 2014; 510:162166.

22 Kitada T, Asakawa S, Hattori N, et al. Mutations in the parkin gene cause autosomal recessive juvenile parkinsonism. $\mathrm{Na}$ ture 1998; 392:605-608.

23 Kazlauskaite A, Kondapalli C, Gourlay R, et al. Parkin is activated by PINK1-dependent phosphorylation of ubiquitin at Ser65. Biochem J 2014; 460:127-139.

24 Sarraf SA, Raman M, Guarani-Pereira V, et al. Landscape of the PARKIN-dependent ubiquitylome in response to mitochondrial depolarization. Nature 2013; 496:372-376.

25 Heo JM, Ordureau A, Paulo JA, Rinehart J, Harper JW.The PINK1-PARKIN mitochondrial ubiquitylation pathway drives a program of OPTN/NDP52 recruitment and TBK1 activation to promote mitophagy. Mol Cell 2015; 60:7-20.

26 Lazarou M, Sliter DA, Kane LA, et al. The ubiquitin kinase PINK1 recruits autophagy receptors to induce mitophagy. $\mathrm{Na}$ ture 2015; 524:309-314.

27 Harding HP, Zhang Y, Zeng H, et al. An integrated stress response regulates amino acid metabolism and resistance to oxidative stress. Mol Cell 2003; 11:619-633.

28 Baker BM, Nargund AM, Sun T, Haynes CM. Protective coupling of mitochondrial function and protein synthesis via the eIF2 $\alpha$ kinase GCN-2. PLoS Genet 2012; 8:e1002760.

29 Shenton D, Smirnova JB, Selley JN, et al. Global translational responses to oxidative stress impact upon multiple levels of protein synthesis. J Biol Chem 2006; 281:29011-29021.

30 Ishimura R, Nagy G, Dotu I, Chuang JH, Ackerman SL. Activation of GCN2 kinase by ribosome stalling links translation elongation with translation initiation. Elife 2016; 5.

31 Dey M, Cao C, Dar AC, et al. Mechanistic link between PKR dimerization, autophosphorylation, and eIF2 $\alpha$ substrate recognition. Cell 2005; 122:901-913.

32 Heo JM, Livnat-Levanon N, Taylor EB, et al. A stress-responsive system for mitochondrial protein degradation. Mol Cell 2010; 40:465-480.

33 Nielson JR, Fredrickson EK, Waller TC, et al. Sterol oxidation mediates stress-responsive Vms1 translocation to mitochondria. Mol Cell 2017; 68:673-685.

34 Izawa T, Park S-H, Zhao L, Hartl FU, Neupert W. Cytosolic protein Vms1 links ribosome quality control to mitochondrial and cellular homeostasis. Cell 2017; 171:890-903.

35 Wrobel L, Topf U, Bragoszewski P, et al. Mistargeted mitochondrial proteins activate a proteostatic response in the cyto- sol. Nature 2015; 524:485-488.

36 Wang X, Chen XJ. A cytosolic network suppressing mitochondria-mediated proteostatic stress and cell death. Nature 2015; 524:481-484.

37 Zhao Q, Wang J, Levichkin IV, Stasinopoulos S, Ryan MT, Hoogenraad NJ. A mitochondrial specific stress response in mammalian cells. EMBO J 2002; 21:4411-4419.

38 Nargund AM, Fiorese CJ, Pellegrino MW, Deng P, Haynes CM. Mitochondrial and nuclear accumulation of the transcription factor ATFS-1 promotes OXPHOS recovery during the UPR(mt). Mol Cell 2015; 58:123-133.

39 Martinus RD, Garth GP, Webster TL, et al. Selective induction of mitochondrial chaperones in response to loss of the mitochondrial genome. Eur J Biochem 1996; 240:98-103.

40 Yoneda T, Benedetti C, Urano F, Clark SG, Harding HP, Ron D. Compartment-specific perturbation of protein handling activates genes encoding mitochondrial chaperones. $J$ Cell Sci 2004; 117:4055-4066.

41 Desjardins P, Frost E, Morais R. Ethidium bromide-induced loss of mitochondrial DNA from primary chicken embryo fibroblasts. Mol Cell Biol 1985; 5:1163-1169.

42 Nargund AM, Pellegrino MW, Fiorese CJ, Baker BM, Haynes CM. Mitochondrial import efficiency of ATFS-1 regulates mitochondrial UPR activation. Science 2012; 337:587-590.

43 Durieux J, Wolff S, Dillin A. The cell-non-autonomous nature of electron transport chain-mediated longevity. Cell 2011; 144:79-91.

44 Kim HE, Grant AR, Simic MS, et al. Lipid biosynthesis coordinates a mitochondrial-to-cytosolic stress response. Cell 2016; 166:1539-1552.

45 Moullan N, Mouchiroud L, Wang X, et al. Tetracyclines disturb mitochondrial function across eukaryotic models: a call for caution in biomedical research. Cell Rep 2015 Mar 10. doi:10.1016/j.celrep.2015.02.034

46 Berendzen KM, Durieux J, Shao LW, et al. Neuroendocrine coordination of mitochondrial stress signaling and proteostasis. Cell 2016; 166:1553-1563.

47 Cai H, Cong WN, Ji S, Rothman S, Maudsley S, Martin B. Metabolic dysfunction in Alzheimer's disease and related neurodegenerative disorders. Curr Alzheimer Res 2012; 9:517.

48 Suomalainen A, Battersby BJ. Mitochondrial diseases: the contribution of organelle stress responses to pathology. Nat Rev Mol Cell Biol 2017 Aug 9. doi:10.1038/nrm.2017.66

49 Suomalainen A, Elo JM, Pietilainen KH, et al. FGF-21 as a biomarker for muscle-manifesting mitochondrial respiratory chain deficiencies: a diagnostic study. Lancet Neurol 2011; 10:806-818.

50 Maruyama R, Shimizu M, Li J, Inoue J, Sato R. Fibroblast growth factor 21 induction by activating transcription factor 4 is regulated through three amino acid response elements in its promoter region. Biosci Biotechnol Biochem 2016; 80:929934.

51 Fusakio ME, Willy JA, Wang Y, et al. Transcription factor ATF4 directs basal and stress-induced gene expression in the unfolded protein response and cholesterol metabolism in the liver. Mol Biol Cell 2016; 27:1536-1551.

52 Dogan SA, Pujol C, Maiti P, et al. Tissue-specific loss of DARS2 activates stress responses independently of respira- 
tory chain deficiency in the heart. Cell Metab 2014; 19:458469.

53 Kambe Y, Miyata A. Potential involvement of the mitochondrial unfolded protein response in depressive-like symptoms in mice. Neurosci Lett 2015; 588:166-171.

54 Khan NA, Nikkanen J, Yatsuga S, et al. mTORC1 regulates mitochondrial integrated stress response and mitochondrial myopathy progression. Cell Metab 2017; 26:419-428.

55 Onyango IG, Dennis J, Khan SM. Mitochondrial dysfunction in Alzheimer's disease and the rationale for bioenergetics based therapies. Aging Dis 2016; 7:201-214.

56 Sorrentino V, Romani M, Mouchiroud L, et al. Enhancing mitochondrial proteostasis reduces amyloid- $\beta$ proteotoxicity. Nature 2017; 552:187-193.

57 Beck JS, Mufson EJ, Counts SE. Evidence for mitochondrial UPR gene activation in familial and sporadic Alzheimer's disease. Curr Alzheimer Res 2016; 13:610-614.

58 Haynes CM, Yang Y, Blais SP, Neubert TA, Ron D. The matrix peptide exporter HAF-1 signals a mitochondrial UPR by activating the transcription factor ZC376.7 in C. elegans. Mol Cell 2010; 37:529-540.

59 Lin YF, Schulz AM, Pellegrino MW, Lu Y, Shaham S, Haynes $\mathrm{CM}$. Maintenance and propagation of a deleterious mitochondrial genome by the mitochondrial unfolded protein response. Nature 2016; 533:416-419.

60 Wright G, Terada K, Yano M, Sergeev I, Mori M. Oxidative stress inhibits the mitochondrial import of preproteins and leads to their degradation. Exp Cell Res 2001; 263:107-117.

61 Rauthan M, Ranji P, Aguilera Pradenas N, Pitot C, Pilon M. The mitochondrial unfolded protein response activator ATFS1 protects cells from inhibition of the mevalonate pathway. Proc Natl Acad Sci USA 2013; 110:5981-5986.

62 Dinur-Mills M, Tal M, Pines O. Dual targeted mitochondrial proteins are characterized by lower MTS parameters and total net charge. PLoS One 2008; 3:e2161.

63 Fukasawa Y, Tsuji J, Fu SC, Tomii K, Horton P, Imai K. MitoFates: improved prediction of mitochondrial targeting sequences and their cleavage sites. Mol Cell Proteomics 2015; 14:1113-1126.

64 Lorch Y, Kornberg RD. Chromatin-remodeling and the initiation of transcription. $Q$ Rev Biophys 2015; 48:465-470.

65 Tian Y, Garcia G, Bian Q, et al. Mitochondrial stress induces chromatin reorganization to promote longevity and UPR(mt). Cell 2016; 165:1197-1208.

66 Merkwirth C, Jovaisaite V, Durieux J, et al. Two conserved histone demethylases regulate mitochondrial stress-induced longevity. Cell 2016; 165:1209-1223.

67 Benedetti C, Haynes CM, Yang Y, Harding HP, Ron D. Ubiquitin-like protein 5 positively regulates chaperone gene expression in the mitochondrial unfolded protein response. Genetics 2006; 174:229-239.

68 Haynes CM, Petrova K, Benedetti C, Yang Y, Ron D. ClpP mediates activation of a mitochondrial unfolded protein response in C. elegans. Dev Cell 2007; 13:467-480.

69 Shao LW, Niu R, Liu Y. Neuropeptide signals cell non-autonomous mitochondrial unfolded protein response. Cell Res 2016; 26:1182-1196.

70 Liu Y, Samuel BS, Breen PC, Ruvkun G. Caenorhabditis elegans pathways that surveil and defend mitochondria. Nature
2014; 508:406-410.

71 Munch C, Harper JW. Mitochondrial unfolded protein response controls matrix pre-RNA processing and translation. Nature 2016; 534:710-713.

72 Quiros PM, Prado MA, Zamboni N, et al. Multi-omics analysis identifies ATF4 as a key regulator of the mitochondrial stress response in mammals. J Cell Biol 2017; 216:20272045.

73 Fiorese CJ, Schulz AM, Lin YF, Rosin N, Pellegrino MW, Haynes CM. The transcription factor ATF5 mediates a mammalian mitochondrial UPR. Curr Biol 2016; 26:2037-2043.

74 Tyynismaa H, Carroll CJ, Raimundo N, et al. Mitochondrial myopathy induces a starvation-like response. Hum Mol Genet 2010; 19:3948-3958.

75 Martinez-Reyes I, Sanchez-Arago M, Cuezva JM. AMPK and GCN2-ATF4 signal the repression of mitochondria in colon cancer cells. Biochem J 2012; 444:249-259.

76 Silva JM, Wong A, Carelli V, Cortopassi GA. Inhibition of mitochondrial function induces an integrated stress response in oligodendroglia. Neurobiol Dis 2009; 34:357-365.

77 Michel S, Canonne M, Arnould T, Renard P. Inhibition of mitochondrial genome expression triggers the activation of CHOP-10 by a cell signaling dependent on the integrated stress response but not the mitochondrial unfolded protein response. Mitochondrion 2015; 21:58-68.

78 Teske BF, Fusakio ME, Zhou D, et al. CHOP induces activating transcription factor 5 (ATF5) to trigger apoptosis in response to perturbations in protein homeostasis. Mol Biol Cell 2013; 24:2477-2490.

79 Zhou D, Palam LR, Jiang L, Narasimhan J, Staschke KA, Wek RC. Phosphorylation of eIF2 directs ATF5 translational control in response to diverse stress conditions. $\mathrm{J}$ Biol Chem 2008; 283:7064-7073.

80 Pakos-Zebrucka K, Koryga I, Mnich K, Ljujic M, Samali A, Gorman AM. The integrated stress response. EMBO Rep 2016; 17:1374-1395.

81 Barbosa C, Peixeiro I, Romao L. Gene expression regulation by upstream open reading frames and human disease. PLoS Genet 2013; 9:e1003529.

82 Young SK, Wek RC. Upstream open reading frames differentially regulate gene-specific translation in the integrated stress response. J Biol Chem 2016; 291:16927-16935.

83 Hinnebusch AG, Ivanov IP, Sonenberg N. Translational control by $5^{\prime}$-untranslated regions of eukaryotic mRNAs. Science 2016; 352:1413-1416.

84 Vattem KM, Wek RC. Reinitiation involving upstream ORFs regulates ATF4 mRNA translation in mammalian cells. Proc Natl Acad Sci USA 2004; 101:11269-11274.

85 Lu PD, Harding HP, Ron D. Translation reinitiation at alternative open reading frames regulates gene expression in an integrated stress response. J Cell Biol 2004; 167:27-33.

86 Gonzalez A, Hall MN. Nutrient sensing and TOR signaling in yeast and mammals. EMBO J 2017; 36:397-408.

87 Magnuson B, Ekim B, Fingar DC. Regulation and function of ribosomal protein $\mathrm{S} 6$ kinase (S6K) within mTOR signalling networks. Biochem J 2012; 441:1-21.

88 Zhao J, Zhai B, Gygi SP, Goldberg AL. mTOR inhibition activates overall protein degradation by the ubiquitin proteasome system as well as by autophagy. Proc Natl Acad Sci USA 
2015; 112:15790-15797.

89 Thoreen CC, Chantranupong L, Keys HR, Wang T, Gray NS, Sabatini DM. A unifying model for mTORC1-mediated regulation of mRNA translation. Nature 2012; 485:109-113.

90 Ben-Sahra I, Hoxhaj G, Ricoult SJH, Asara JM, Manning BD. mTORC1 induces purine synthesis through control of the mitochondrial tetrahydrofolate cycle. Science 2016; 351:728733.

91 Robinson BH, Petrova-Benedict R, Buncic JR, Wallace DC. Nonviability of cells with oxidative defects in galactose medium: a screening test for affected patient fibroblasts. Biochem Med Metab Biol 1992; 48:122-126.

92 Bao XR, Ong SE, Goldberger O, et al. Mitochondrial dysfunction remodels one-carbon metabolism in human cells. Elife 2016; 5.

93 Ducker GS, Rabinowitz JD. One-carbon metabolism in health and disease. Cell Metab 2017; 25:27-42.

94 Ben-Meir A, Yahalomi S, Moshe B, Shufaro Y, Reubinoff B, Saada A. Coenzyme Q-dependent mitochondrial respiratory chain activity in granulosa cells is reduced with aging. Fertil Steril 2015; 104:724-727.

95 Das KC, Muniyappa H. Age-dependent mitochondrial energy dynamics in the mice heart: role of superoxide dismutase-2. Exp Gerontol 2013; 48:947-959.

96 Hughes AL, Gottschling DE. An early age increase in vacuolar $\mathrm{pH}$ limits mitochondrial function and lifespan in yeast. Nature 2012; 492:261-265.

97 Gruber J, Ng LF, Fong S, et al. Mitochondrial changes in ageing Caenorhabditis elegans — what do we learn from superoxide dismutase knockouts? PLoS One 2011; 6:e19444.

98 Copeland JM, Cho J, Lo T Jr, et al. Extension of Drosophila life span by RNAi of the mitochondrial respiratory chain. Curr Biol 2009; 19:1591-1598.

99 Rea SL, Ventura N, Johnson TE. Relationship between mitochondrial electron transport chain dysfunction, development, and life extension in Caenorhabditis elegans. PLoS Biol 2007; 5:e259.

100 Dillin A, Hsu AL, Arantes-Oliveira N, et al. Rates of behavior and aging specified by mitochondrial function during development. Science 2002; 298:2398-2401.

101 Felkai S, Ewbank JJ, Lemieux J, Labbe JC, Brown GG, Hekimi S. CLK-1 controls respiration, behavior and aging in the nematode Caenorhabditis elegans. EMBO J 1999; 18:17831792.

102 Liu X, Jiang N, Hughes B, Bigras E, Shoubridge E, Hekimi S. Evolutionary conservation of the clk-1-dependent mechanism of longevity: loss of mclk1 increases cellular fitness and lifespan in mice. Genes Dev 2005; 19:2424-2434.

103 Houtkooper RH, Mouchiroud L, Ryu D, et al. Mitonuclear protein imbalance as a conserved longevity mechanism. $\mathrm{Na}$ ture 2013; 497:451-457.

104 Pellegrino MW, Nargund AM, Kirienko NV, Gillis R, Fiorese CJ, Haynes CM. Mitochondrial UPR-regulated innate immunity provides resistance to pathogen infection. Nature 2014; 516:414-417.

105 Garcia-Prat L, Martinez-Vicente M, Perdiguero E, et al. Autophagy maintains stemness by preventing senescence. Nature 2016; 529:37-42.

106 Schiavi A, Maglioni S, Palikaras K, et al. Iron-starvation-in- duced mitophagy mediates lifespan extension upon mitochondrial stress in C. elegans. Curr Biol 2015; 25:1810-1822.

107 Rana A, Rera M, Walker DW. Parkin overexpression during aging reduces proteotoxicity, alters mitochondrial dynamics, and extends lifespan. Proc Natl Acad Sci USA 2013; 110:8638-8643.

108 Palikaras K, Lionaki E, Tavernarakis N. Coordination of mitophagy and mitochondrial biogenesis during ageing in $C$. elegans. Nature 2015; 521:525-528.

109 Conboy IM, Conboy MJ, Wagers AJ, Girma ER, Weissman IL, Rando TA. Rejuvenation of aged progenitor cells by exposure to a young systemic environment. Nature 2005; 433:760764 .

110 Takahashi K, Yamanaka S. Induction of pluripotent stem cells from mouse embryonic and adult fibroblast cultures by defined factors. Cell 2006; 126:663-676.

111 Honoki K. Preventing aging with stem cell rejuvenation: Feasible or infeasible? World J Stem Cells 2017; 9:1-8.

112 Ito K, Suda T. Metabolic requirements for the maintenance of self-renewing stem cells. Nat Rev Mol Cell Biol 2014; 15:243256.

113 Seita J, Weissman IL. Hematopoietic stem cell: self-renewal versus differentiation. Wiley Interdiscip Rev Syst Biol Med 2010; 2:640-653.

114 Oh J, Lee YD, Wagers AJ. Stem cell aging: mechanisms, regulators and therapeutic opportunities. Nat Med 2014; 20:870880.

115 Simsek T, Kocabas F, Zheng J, et al. The distinct metabolic profile of hematopoietic stem cells reflects their location in a hypoxic niche. Cell Stem Cell 2010; 7:380-390.

116 Ahlqvist KJ, Suomalainen A, Hamalainen RH. Stem cells, mitochondria and aging. Biochim Biophys Acta 2015; 1847:1380-1386.

117 Norddahl GL, Pronk CJ, Wahlestedt M, et al. Accumulating mitochondrial DNA mutations drive premature hematopoietic aging phenotypes distinct from physiological stem cell aging. Cell Stem Cell 2011; 8:499-510.

118 Ahlqvist KJ, Hamalainen RH, Yatsuga S, et al. Somatic progenitor cell vulnerability to mitochondrial DNA mutagenesis underlies progeroid phenotypes in Polg mutator mice. Cell Metab 2012; 15:100-109.

119 Trifunovic A, Wredenberg A, Falkenberg M, et al. Premature ageing in mice expressing defective mitochondrial DNA polymerase. Nature 2004; 429:417-423.

120 Fox RG, Magness S, Kujoth GC, Prolla TA, Maeda N. Mitochondrial DNA polymerase editing mutation, PolgD257A, disturbs stem-progenitor cell cycling in the small intestine and restricts excess fat absorption. Am J Physiol Gastrointest Liver Physiol 2012; 302:G914-G924.

121 Chen ML, Logan TD, Hochberg ML, et al. Erythroid dysplasia, megaloblastic anemia, and impaired lymphopoiesis arising from mitochondrial dysfunction. Blood 2009; 114:40454053.

122 Mohrin M, Shin J, Liu Y, et al. Stem cell aging. A mitochondrial UPR-mediated metabolic checkpoint regulates hematopoietic stem cell aging. Science 2015; 347:1374-1377.

123 Miller FJ, Rosenfeldt FL, Zhang C, Linnane AW, Nagley P. Precise determination of mitochondrial DNA copy number in human skeletal and cardiac muscle by a PCR-based assay: 
lack of change of copy number with age. Nucleic Acids Res 2003; 31:e61.

124 Kukat C, Wurm CA, Spahr H, Falkenberg M, Larsson NG, Jakobs S. Super-resolution microscopy reveals that mammalian mitochondrial nucleoids have a uniform size and frequently contain a single copy of mtDNA. Proc Natl Acad Sci USA 2011; 108:13534-13539.

125 Gaziev AI, Abdullaev S, Podlutsky A. Mitochondrial function and mitochondrial DNA maintenance with advancing age. Biogerontology 2014; 15:417-438.

126 Wallace DC, Chalkia D. Mitochondrial DNA genetics and the heteroplasmy conundrum in evolution and disease. Cold Spring Harb Perspect Biol 2013; 5:a021220.

127 Stewart JB, Chinnery PF. The dynamics of mitochondrial DNA heteroplasmy: implications for human health and disease. Nat Rev Genet 2015; 16:530-542.

128 Payne BA, Wilson IJ, Yu-Wai-Man P, et al. Universal heteroplasmy of human mitochondrial DNA. Hum Mol Genet 2013; 22:384-390.

129 Wallace DC. A mitochondrial paradigm of metabolic and degenerative diseases, aging, and cancer: a dawn for evolutionary medicine. Annu Rev Genet 2005; 39:359-407.

130 Kauppila TES, Kauppila JHK, Larsson NG. Mammalian mitochondria and aging: An update. Cell Metab 2017; 25:57-71.

131 Gitschlag BL, Kirby CS, Samuels DC, Gangula RD, Mallal SA, Patel MR. Homeostatic responses regulate selfish mitochondrial genome dynamics in C. elegans. Cell Metab 2016; 24:91-103.

132 Valenci I, Yonai L, Bar-Yaacov D, Mishmar D, Ben-Zvi A. Parkin modulates heteroplasmy of truncated mtDNA in Caenorhabditis elegans. Mitochondrion 2015; 20:64-70.

133 Suen DF, Narendra DP, Tanaka A, Manfredi G, Youle RJ. Parkin overexpression selects against a deleterious mtDNA mutation in heteroplasmic cybrid cells. Proc Natl Acad Sci USA 2010; 107:11835-11840.
134 Tyynismaa H, Mjosund KP, Wanrooij S, et al. Mutant mitochondrial helicase Twinkle causes multiple mtDNA deletions and a late-onset mitochondrial disease in mice. Proc Natl Acad Sci USA 2005; 102:17687-17692.

135 Gilkerson RW, De Vries RL, Lebot P, et al. Mitochondrial autophagy in cells with mtDNA mutations results from synergistic loss of transmembrane potential and mTORC1 inhibition. Hum Mol Genet 2012; 21:978-990.

136 Jin SM, Youle RJ. The accumulation of misfolded proteins in the mitochondrial matrix is sensed by PINK1 to induce PARK2/Parkin-mediated mitophagy of polarized mitochondria. Autophagy 2013; 9:1750-1757.

137 Gerbeth C, Schmidt O, Rao S, et al. Glucose-induced regulation of protein import receptor Tom 22 by cytosolic and mitochondria-bound kinases. Cell Metab 2013; 18:578-587.

138 Harbauer AB, Zahedi RP, Sickmann A, Pfanner N, Meisinger $\mathrm{C}$. The protein import machinery of mitochondria - a regulatory hub in metabolism, stress, and disease. Cell Metab 2014; 19:357-372.

139 Schmidt O, Harbauer AB, Rao S, et al. Regulation of mitochondrial protein import by cytosolic kinases. Cell 2011; 144:227-239.

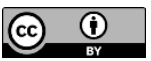

This work is licensed under a Creative Commons Attribution 4.0 Unported License. The images or other third party material in this article are included in the article's Creative Commons license, unless indicated otherwise in the credit line; if the material is not included under the Creative Commons license, users will need to obtain permission from the license holder to reproduce the material. To view a copy of this license, visit http:// creativecommons.org/licenses/by/4.0/

C The Author(s) 2018 